\title{
Biotoxicité hématologique du sulfate de cadmium chez les rats Wistar
}

\author{
Vandjiguiba DIABY ${ }^{1,2^{*}}$, Adou Francis YAPO $^{2}$ Arsène Mousan ADON $^{1}$, \\ Houphouet Félix YAPI ${ }^{2}$, Allico Joseph DJAMA ${ }^{2,3}$ et Mireille DOSSO ${ }^{1,3}$ \\ ${ }^{1}$ Unité de Biologie Cellulaire, Institut Pasteur de Cote d'Ivoire, 01 BP 490 Abidjan 01, Côte d'Ivoire. \\ ${ }^{2}$ Laboratoire de Pharmacodynamie-Biochimique, UFR Biosciences, Université Felix Houphouet Boigny - \\ Abidjan, 22 BP 582 Abidjan 22, Côte d'Ivoire. \\ ${ }^{3}$ Département de Biochimie Médicale et Fondamentale Institut Pasteur de Cote d'Ivoire, \\ 01 BP 490 Abidjan 01, Cote d'Ivoire. \\ *Auteur correspondant ; E-mail : vandjiguiba@yahoo.fr
}

\section{RESUME}

L'appréciation hématologique chez les animaux est d'un intérêt capital pour définir le diagnostic de nombreuses maladies. L'altération des paramètres (les globules blancs et les globules rouges) est un indicateur d'exposition précoce aux toxiques. Six (6) lots de rats Wistar mâles et six (6) lots femelles ont été constitués ; chaque lot étant composé de cinq (5) rats. Ces rats ont été contaminés au sulfate de cadmium par gavage journalier durant 30 jours à différentes doses, à savoir $4 ; 5 ; 6,66 ; 10$ et $20 \mathrm{mg} / \mathrm{kg}$ de poids corporel $(\mathrm{pc})$ correspondant respectivement à $1 / 50^{\text {ième }}, 1 / 40^{\text {ième }} 1 / 30^{\text {ième }} 1 / 20^{\text {ième }} 1 / 10^{\text {ième }}$ de la $\mathrm{DL}_{50}$. Les rats étaient pesés une fois par semaine. A la fin de l'expérience, le sang total a été recueilli dans des tubes à EDTA après avoir sacrifié les rats. Le sang a servi à l'hémogramme avec le Sysmex XN-1000. Les résultats ont montré que le cadmium a entrainé une augmentation des globules blancs, une diminution des globules rouges, de l'hémoglobine et des hématocrites chez les mâles comme chez les femelles par rapport au témoin. Le cadmium a entrainé une inflammation caractérisée par l'augmentation des globules blancs et une anémie.

(C) 2016 International Formulae Group. All rights reserved.

Mots clés : Sulfate de cadmium, hémogramme, rat, anémie.

\section{Hematological biotoxicity of cadmium sulphate in the Wistar rats}

\begin{abstract}
The hematological evaluation in animals is of paramount interest to define the diagnosis of many diseases. The alteration of these parameters (white blood cell and red blood cell) is an early indicator of exposure to toxics. Six (6) male Wistar rats groups and six (6) female groups were formed; each group being composed of five (5) rats. These rats were contaminated with cadmium sulfate by daily gavage for 30 days at different doses including $4 ; 5 ; 6.66 ; 10$ and $20 \mathrm{mg} / \mathrm{kg}$ body weight corresponding respectively to $1 / 50^{\text {th }}, 1 / 40^{\text {th }}$, $1 / 30^{\text {th }}, 1 / 20^{\text {th }}, 1 / 10^{\text {th }}$ of the $\mathrm{LD}_{50}$. The rats were weighed weekly. At the end of the experiment, whole blood was used for blood count. The results obtained showed that the $\mathrm{LD}_{50}$ of cadmium sulfate was $200 \mathrm{mg} / \mathrm{kg}$ of body weight. Cadmium induced an increase in white blood cells, a decrease in red blood cells, hemoglobin and hematocrit in males as well as in females compared to control. Cadmium created an inflammation characterized by an increase in the white blood cells and anemia.

(C) 2016 International Formulae Group. All rights reserved.
\end{abstract}

Keywords: Sulfate cadmium, hemogram, rat, anemia. 


\section{INTRODUCTION}

La présence d'éléments traces dans la fiente représente une contrainte majeure à son utilisation en agriculture. La fiente de volaille constitue une source d'enrichissement des sols maraîchers de la ville d'Abidjan en métaux traces (Kouassi et al., 2008). Selon une étude menée par Apata et al. (2014), ces fientes contiennent une très forte concentration en cadmium. La présence de $\mathrm{Cd}$ dans les fientes traduit le niveau de contamination dans les feuilles de l'épinard (Spinacia oleracea) et l'exposition des poulets à une alimentation polluée au cadmium. Les légumes et les céréales sont donc les sources principales de cadmium (Koffi et al., 2014) surtout dans les feuilles des gombos, corète potagère, épinard et aubergine constatées par Nantarie et al. (2015). Ces légumes constituent l'alimentation des ivoiriens. L'ingestion d'aliment et d'eau sont les voies de contamination au cadmium (Amir et al., 2014). A travers cette alimentation, le cadmium s'accumule dans le corps et cause beaucoup de maladies (Hounkpatin et al., 2013). Ainsi, l'exposition chronique au cadmium à faible concentration provoque la défaillance rénale, l'ostéoporose, un dérèglement de la pression sanguine et un risque élevé de cancer (Satarug et Moore., 2004). Et une exposition excessive peut entrainer la mort (Othumpangat et al., 2005; Hounkpatin et al., 2013). Le cadmium ingéré atteint les tissus via le sang (Hounkpatin et al., 2013). Ainsi, la détermination des paramètres hématologique des animaux est importante pour établir le diagnostic de nombreuses maladies. L'altération de ces paramètres hématologiques (les globules blancs et les globules rouges) est un indicateur d'exposition précoce aux toxiques qui affectent les tissus (Vinodini et al., 2015). A cet égard, la présente étude à pour objectif d'évaluer la toxicité du sulfate de cadmium sur la perturbation du tissu sanguin chez les rats Wistar.

\section{MATERIEL ET METHODES Matériel}

Le matériel biologique est constitué de sang total des rats mâles et femelles de souche
Wistar pesant $106 \pm 6 \mathrm{~g}$ et âgés de 8 à 12 semaines. Les rats ont été placés dans des cages garnies de litières de copeau de bois dont le renouvellement se faisait deux fois par semaine. Ils ont été acclimatés deux semaines avant le début de la manipulation.

Le sulfate de cadmium utilisé pour les essais était en sel déshydraté, de marque MERCK et dont le numéro de série est 1.02027.0100.

\section{Méthode}

Pour l'expérience, chaque lot est constitué d'animaux de même sexe et de poids sensiblement égaux. Les animaux ont été marqués à la queue par lot et à l'intérieur de chaque lot afin de les identifier au cours de l'expérimentation. Douze (12) lots de cinq (5) rats ont été constitués dont 6 lots mâles et 6 lots de femelles. Lot 1 (mâles) et lot 1 (femelles) ont constitué les lots témoins où les animaux recevaient de l'eau distillée par gavage ( $1 \mathrm{ml} /$ Jour). Les lots $2,3,4,5$ et 6 de chaque sexe ont reçu par gavage respectivement $1 / 50^{\text {ième }}, 1 / 40^{\text {ième }}, 1 / 30^{\text {ième }}$, soit $1 / 20^{\text {ième }}$ et $1 / 10^{\text {ième }}$ de la $D_{50}(200 \mathrm{mg} / \mathrm{kg}$ pc) de la solution de sulfate de cadmium qui sont respectivement $4 ; 5 ; 6,66 ; 10$ et 20 $\mathrm{mg} / \mathrm{kg}$ de poids corporel (pc). La durée de traitement des rats au sulfate de cadmium était de 30 Jours (Layachi et Kechrid, 2012). Et le volume administré était de $1 \mathrm{ml}$ par jour chaque matin pendant la durée de traitement. La méthode OCDE 423 a été utilisée pour déterminer la DL50 du sulfate de cadmium. Les rats ont été sacrifiés par décapitation après la période d'exposition (30 Jours) au sulfate de cadmium. Le sang total de chaque rat a été recueilli immédiatement dans des tubes EDTA pour la numération de la formule sanguine à l'Institut Pasteur d'Abidjan (Côte d'Ivoire) avec le Sysmex XN-1000 dans moins de 4 heures.

\section{Analyses statistiques}

Le logiciel G raphPad.Prism.V5.01 a été utilisé pour l'analyse statistique des résultats et le tracé des courbes. Les données ont été analysées avec ANOVA One-Way. Le test $t$ non paramétrique de Dunnett a été utilisé pour la comparaison de la variance des témoins 
avec celle des autres groupes. La différence entre deux variances était significative si $\mathrm{p}<0,05$.

\section{RESULTATS}

Effets du sulfate de cadmium sur les paramètres de la lignée érythrocytaire (Globules rouges, hémoglobine, hématocrite, MCV (Mean corpuscular volume), MCH (Mean corpuscular hemoglobin), MCHC (Mean corpuscular hemoglobin concentration))

Les valeurs des globules rouges, de l'hémoglobine, des hématocrites (Tableau I) ont été significativement diminuées $(\mathrm{p}<0,001)$ chez les femelles comme chez les mâles. Chez les mâles, la diminution des globules rouges est plus significative à la dose $5 \mathrm{mg} / \mathrm{kg}(7,06 \pm$ $\left.0,97.10^{6} / \mu 1, \mathrm{p}<0,001\right)$ et chez les femelles à la dose $20 \mathrm{mg} / \mathrm{kg}\left(7,38 \pm 0,45.10^{6} / \mu \mathrm{l}, \mathrm{p}<0,001\right)$ comparativement aux témoins (mâles: $8,75 \pm$

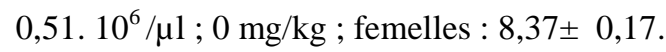
$10 \% / \mu 1 ; 0 \mathrm{mg} / \mathrm{kg})$.

Pour l'hémoglobine, cette diminution est plus significative à la dose $20 \mathrm{mg} / \mathrm{kg}$ (mâles: $12 \pm 2 \mathrm{~g} / \mathrm{dl}, \mathrm{p}<0,001$; femelles: $14 \pm 1$ $\mathrm{g} / \mathrm{dl}, \mathrm{p}<0,001)$ comparativement aux témoins (mâles: $16 \pm 1 \mathrm{~g} / \mathrm{dl} ; 0 \mathrm{mg} / \mathrm{kg}$; femelles : $16 \pm 1$ $\mathrm{g} / \mathrm{dl} ; 0 \mathrm{mg} / \mathrm{kg}$ ). Cependant chez les femelles, cette diminution est également significative aux autres doses $(4 ; 5 ; 6,66$ et $10 \mathrm{mg} / \mathrm{kg}$ ) (Tableau 1).

Quant à l'hématocrite, la diminution constatée est significative à la dose $5 \mathrm{mg} / \mathrm{kg}$ chez les mâles $(38 \pm 15 \% ; \mathrm{p}<0,05)$ et 20 $\mathrm{mg} / \mathrm{kg}$ chez les femelles $(41 . \pm 5 \% ; \mathrm{p}<0,001)$

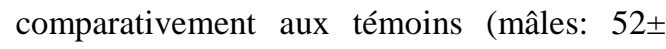
4\%; $0 \mathrm{mg} / \mathrm{kg}$; femelles : $50 \pm 4 \%$; $0 \mathrm{mg} / \mathrm{kg}$ ).

Les $\mathrm{MCV}$ et des $\mathrm{MCH}$ ont significativement diminuées $(\mathrm{p}<0,001)$ chez les femelles. Par contre, les valeurs de ces éléments figurés du sang n'ont pas connu de variation significative chez les mâles (Tableau 1).
L'effet du sulfate de cadmium sur la numération glomérulaire n'est pas dosedépendant.

Effets du sulfate de cadmium sur les paramètres de la lignée leucocytaire (Globules blancs, neutrophiles, monocytes, lymphocytes)

Les globules blancs ont subi une augmentation significative à partir des concentrations supérieures à $4 \mathrm{mg} / \mathrm{kg}$ pc aussi bien chez les rats mâles que chez les rats femelles comparativement aux témoins (mâles: 7000 $\pm 1301 \mu 1,0 \mathrm{mg} / \mathrm{kg}$; femelles : $6290 \pm 891 \mu 1,0 \mathrm{mg} / \mathrm{kg}$ ) (Tableau 1). Cette augmentation est très importante pour les lots à fortes doses (mâles: $14965 \pm 559 \mu 1$,

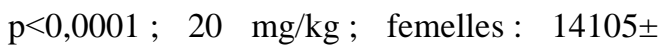
$3967 \mu 1, \mathrm{p}<0,0001 ; 20 \mathrm{mg} / \mathrm{kg}$ ) et est dosedépendant chez les mâles et non chez les femelles. Cette augmentation concerne également les lymphocytes (mâles : $6095 \pm$ $728.10^{3} / \mu 1, \mathrm{p}<0,0001$; femelles : $5945 \pm 841$. $10^{3} / \mu 1, \mathrm{p}<0,0001$ ), les monocytes (mâles :

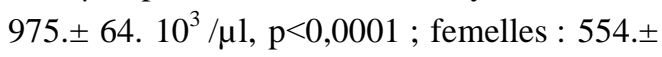
175. $\left.10^{3} / \mu 1, \mathrm{p}<0,0001\right)$ et les neutrophiles (mâles : $\quad 9080 . \pm 1018.10^{3} / \mu 1, \quad \mathrm{p}<0,0001$; femelles : 8609. $\left.\pm 2746.10^{3} / \mu 1, \mathrm{p}<0,0001\right)$.

Effets du sulfate de cadmium sur les plaquettes sanguines

Le cadmium a entrainé une diminution des plaquettes chez les mâles comparativement aux témoins (Tableau 1). Cette diminution est plus significative à la dose $5 \mathrm{mg} / \mathrm{kg}$ (mâles: $689 \pm 448 \mu \mathrm{l}$, $\mathrm{p}<0,001$ ) et à la dose $10 \mathrm{mg} / \mathrm{kg}$ (mâles: 763 $\pm 195 \mu 1, \quad \mathrm{p}<0,001) \quad$ comparativement aux témoins (mâles: $1255 \pm 210 \mu \mathrm{l}$ ).

Contrairement aux femelles, la diminution constatée est non significative comparativement aux témoins. 
V. DIABY et al. / Int. J. Biol. Chem. Sci. 10(4): 1765-1772, 2016

Tableau 1: Effet du cadmium sur la Formule globulaire chez les rats.

\begin{tabular}{|c|c|c|c|c|c|c|c|}
\hline Paramètres & Sexes & LOT 1 (Témoin) & LOT 2 (4 mg/kg) & LOT 3 (5 mg/kg) & LOT4 $(6,66 \mathrm{mg} / \mathrm{kg})$ & LOT5 (10 mg/kg) & LOT6 (20 mg/kg) \\
\hline \multirow[t]{2}{*}{ Globules Blancs $\left(10^{3} / \mu 1\right)$} & Mâle & $7000 \pm 1301$ & $9175 \pm 92$ & $9840 \pm 1230 *$ & $11645 \pm 3132 * * *$ & $14037 \pm 868^{* * *}$ & $14965 \pm 559 * * *$ \\
\hline & Femelle & $6290 \pm 891$ & $8790 \pm 212$ & $8090 . \pm 778$ & $9455 \pm 403$ & $11680 \pm 3790 * *$ & $14105 \pm 3967 * * *$ \\
\hline \multirow[t]{2}{*}{ P. Neutrophiles $\left(10^{3} / \mu 1\right)$} & Mâle & $6230 \pm 608$ & $6560 . \pm 641 * * *$ & $6450 . \pm 1188^{* * *}$ & $8805 \pm 474 * * *$ & $9080 . \pm 1018 * * *$ & $7320 . \pm 1004$ \\
\hline & Femelle & $5195 \pm 983$ & $5450 \pm 468$ & $7600 . \pm 14^{*}$ & $5430 \pm 106$ & $5887 \pm 490$ & $8609 . \pm 2746^{* * *}$ \\
\hline \multirow[t]{2}{*}{ Monocytes $\left(10^{3} / \mu 1\right)$} & Mâle & $435 . \pm 35$ & $243 \pm 45^{* *}$ & $183 . \pm 29 * * *$ & $720 \pm 156^{* * *}$ & $500 . \pm 99$ & $975 . \pm 64 * * *$ \\
\hline & Femelle & $123 \pm 49$ & $325 \pm 35 * *$ & $250 . \pm 56$ & $423 \pm 31 * * *$ & $460 . \pm 14 * * *$ & $554 . \pm 175 * * *$ \\
\hline \multirow[t]{3}{*}{ Lymphocytes $\left(10^{3} / \mu 1\right)$} & Mâle & $2240 \pm 823$ & $2067 . \pm 159$ & $2915 . \pm 332$ & $6095 \pm 728 * * *$ & $4810 \pm 792 * * *$ & $4350 \pm 961 * * *$ \\
\hline & Femelle & $2810 \pm 396$ & $4507 \pm 782 *$ & $3867 \pm 480$ & $5945 \pm 841 * * *$ & $3483 . \pm 1369$ & $3729 . \pm 992$ \\
\hline & Mâle & $8,75 \pm 0,51$ & $8,21 \pm 0,21$ & $7,06 \pm 0,97 * *$ & $7,54 \pm 0,81$ & $7,70 \pm 0,98$ & $7,65 \pm 0,66$ \\
\hline \multirow[t]{2}{*}{ Globules Rouges $\left(10^{6} / \mu \mathrm{l}\right)$} & Femelle & $8,37 \pm 0,17$ & $8,32 \pm 0,11$ & $8,45 \pm 0,37$ & $8,07 \pm 0,26$ & $8,50 \pm 0,71$ & $7,38 \pm 0,45^{* *}$ \\
\hline & Mâle & $16 \pm 1$ & $14 \pm 2$ & $13 \pm 3$ & $15 \pm 0$ & $14 \pm 1$ & $12 \pm 2 * *$ \\
\hline Hémoglobine (g/dl) & Femelle & $16 \pm 1$ & $14 \pm 0 * *$ & $14 \pm 1 * *$ & $14 \pm 1 * *$ & $14 \pm 1 * *$ & $14 \pm 1 * *$ \\
\hline \multirow{2}{*}{ Hématocrite $(\%)$} & Mâle & $52 \pm 4$ & $51 \pm 6$ & $38 \pm 15^{*}$ & $53 \pm 3$ & $49 \pm 5$ & $50 . \pm 1$ \\
\hline & Femelle & $50 \pm 4$ & $48 \pm 3 *$ & $49 \pm 3$ & $47 \pm 3$ & $49 \pm 6$ & $41 . \pm 5 * *$ \\
\hline \multirow{2}{*}{ Plaquettes $\left(10^{3} / \mu 1\right)$} & Mâle & $1255 \pm 210$ & $1057 \pm 73$ & $689 \pm 448 * *$ & $1076 \pm 95$ & $763 \pm 195 * *$ & $1086 \pm 35$ \\
\hline & Femelle & $876 \pm 80$ & $842 \pm 286$ & $860 \pm 116$ & $846 \pm 149$ & $1006 \pm 262$ & $818 \pm 57$ \\
\hline \multirow[t]{2}{*}{ MCV (fl) } & Mâle & $60 \pm 1$ & $59 \pm 2$ & $61 \pm 5$ & $58 \pm 2$ & $59 \pm 5$ & $55 \pm 3$ \\
\hline & Femelle & $62 \pm 2$ & $59 \pm 2$ & $59 \pm 1$ & $59 \pm 0$ & $62 \pm 6$ & $56 \pm 1 * *$ \\
\hline \multirow[t]{2}{*}{$\mathrm{MCH}(\mathrm{pg})$} & Mâle & $17 \pm 1$ & $18 \pm 1$ & $18 \pm 4$ & $19 \pm 4$ & $17 \pm 1$ & $17 \pm 4$ \\
\hline & Femelle & $19 \pm 1$ & $17 \pm 1 * *$ & $17 \pm 1 * *$ & $17 \pm 1 * *$ & $16 \pm 0 * * *$ & $17 \pm 1 * *$ \\
\hline \multirow{2}{*}{$\mathrm{MCHC}(\mathrm{g} / \mathrm{dl})$} & Mâle & $29 \pm 1$ & $30 \pm 2$ & $29 \pm 4$ & $29 \pm 1$ & $30 \pm 2$ & $31 \pm 5$ \\
\hline & Femelle & $30 \pm 1$ & $29 \pm 2$ & $30 \pm 1$ & $29 \pm 2$ & $30 \pm 2$ & $31 \pm 2$ \\
\hline
\end{tabular}




\section{DISCUSSION}

Le mécanisme de toxicité du cadmium n'est pas complètement élucidé (Milovanovic et al., 2009). Toutefois, la rate, le foie, et la moelle osseuse qui sont des organes erythropoietiques sont la cible du cadmium (Oluwafemi et al., 2014). Ainsi, les paramètres hématologiques sont utilisés comme indicateur précoce de la toxicité des polluants sur ces tissus (Vinodini et al., 2015). Les résultats obtenus lors des travaux ont montré une diminution des globules rouges, de l'hémoglobine et des hématocrites à la fois chez les femelles comme chez les mâles de manière significative par rapport aux témoins. Même constat fait par Fawzia et al.( 2014) chez les rats femelles gestante contaminées au chlorure de cadmium. Cette diminution est considérée comme une anémie rapportée par Horiguchi (2007). L'anémie est une importante expression de la toxicité du cadmium. Elle pourrait donc s'expliquer par une augmentation de la destruction des globules rouges et en même temps une diminution de leurs synthèses. En effet, les métaux, en s'accumulant dans la rate, le foie et les reins, inhibent l'activité erythropoietique en endommageant la synthèse de l'érythropoïétine qui est une hormone secrétée par les reins dont le rôle est la stimulation des globules rouges (Oluwafemi et al., 2014). Le cadmium perturbe donc le système hématopoïétique (Shim et al., 2008; Fahim et al., 2012). Ainsi donc, pour Hounkpatin et al. (2013), le cadmium cause des désordres sanguins. Dans leur étude sur l'effet combiné de la toxicité du cadmium et du mercure, ils ont constaté une diminution de l'hémoglobine et une diminution en MCHC symbolisant une anémie hypochrome et macrocytaire. Dans le même sens, Anju (2012) suggère une déficience en fer qui pourrait contribuer à l'anémie chez les poulets traités au cadmium lors de son étude. Les résultats des études antérieures sont similaires à ceux obtenus dans cette étude. En effet, les résultats obtenus corroborent avec ceux de Oluwafemi et al. (2014) sur le traitement de l'intoxication au cadmium par Irvingia donnant une diminution de l'hémoglobine, de l'hématocrite, des globules rouges, $\mathrm{MCV}, \mathrm{MCH}$ et $\mathrm{MCHC}$ chez les rats responsable de l'anémie. Les travaux d'Anju (2012) également sont similaires aux résultats obtenus. Au niveau de la formule leucocytaire, les résultats des travaux réalisés, ont également montré une augmentation du taux des globules blancs de manière significative comparativement au témoin. L'augmentation de ces cellules sanguines témoigne d'un état inflammatoire général (Fahim et al., 2012) due aux dommages causées par la toxicité du cadmium (Alex et Lawrence, 2013). Ce qui pourrait s'expliquer par la stimulation du système immunitaire entraînant ces augmentations (Alex et Lawrence, 2013). Cette augmentation de manière considérable caractérise une leucémie selon une étude menée sur des rats contaminés par ingestion au chlorure de cadmium (IARC, 1993). Ces résultats corroborent avec ceux de Jelena et al. (2014) qui ont constaté une augmentation significative des leucocytes du sang périphérique, des neutrophiles par rapport au témoin sur des rats traités par injection intra-peritonien à $1 \mathrm{mg} / \mathrm{kg}$ pendant 48 heures au Cadmium. Aussi, Gabol et al. (2014) et Borane (2013) ont-ils constaté une augmentation des globules blancs et une diminution des globules rouges après administration du cadmium aux doses respectives de $20 \mu \mathrm{g} / \mathrm{kg}$ aux poulets domestiques et de 1,248 ppm aux poissons. Egalement chez les amphibiens en fonction des doses d'expositions $(0,25 ; 0,50 ; 1,00$ et 2,00 mg/l), Alex et Lawrence (2013) ont constaté une augmentation des leucocytes.

Cependant, l'effet du cadmium constaté n'est pas dose-dépendant. Ce qui s'explique par le fait que le taux d'accumulation des métaux lourds chez les animaux varie en fonction des espèces et en fonction des individus dans la population. Il dépend du sexe, de l'âge, de la taille et de l'alimentation (Bedii et ENGIN., 2005; Haki K. et al., 2005).

Ainsi, les changements physicomorphologiques du sang dus aux effets toxiques du cadmium sont un indicateur de 
l'altération de la qualité de l'environnement et peuvent servir comme bio-indicateur d'exposition aux toxiques dans le cadre d'une étude d'évaluation environnementale (Padmanaban et Mohan, 2013).

\section{Conclusion}

Le sulfate de cadmium a entraîné une perturbation hématologique voire un désordre sanguin aboutissant à une infection et à une anémie chez les rats exposés. Ces travaux ainsi réalisés peuvent servir d'outil pour l'évaluation de la qualité environnementale par l'utilisation des rats comme bio détecteur d'exposition aux métaux lourds dans le cadre d'une étude d'évaluation environnementale. Cela passe par le dosage du cadmium dans le sang des rats sauvages et l'observation des paramètres hématologiques.

\section{CONTRIBUTIONS DES AUTEURS}

Tous les auteurs ont contribué efficacement à la réalisation de ce travail à quelque niveau que ce soit. C'est ainsi que DV a réalisé les expérimentations sous la supervision de YAF qui a dirigé le travail. AAM a contribué à travers la recherche du métal lourd et à l'analyse des résultats. Ces trois premiers auteurs ont analysé les résultats et rédigé l'article. Quant à YHF, DAJ et DM, ils ont, de par l'entremise de leur service, certifié les résultats et contribué à l'amélioration de la version finale de l'article.

\section{CONFLIT D'INTERETS}

Les auteurs déclarent qu'il n'y a pas de conflit d'intérêts.

\section{REMERCIEMENTS}

Les auteurs remercient, le Département de Biochimie Médicale et Fondamentale de l'Institut Pasteur de Cote d'Ivoire pour la réalisation des tests biologiques. Aussi, voudraient-ils remercier Département de SVT de l'ENS de CI pour avoir permis l'acclimatation des rats et les expérimentations dans son animalerie

\section{REFERENCES}

Alex AE, Lawrence IE. 2013. The effects of sub lethal concentrations of cadmium on haematological indices of two amphibians. ICMBPS, 4(5):216-219.

Amir W, Jahanzaib A, Farhat I, Ashif S, Zahid M, Ghulam M. 2014. Pollution Status of Pakistan: A Retrospective Review on Heavy Metal Contamination of Water, Soil, and Vegetables. BioMed Research International, 29p. http://dx.doi.org/10.1155/2014/813206

Apata A, Koffi RN, Touré N, Alui K A, YaoKouamé A. 2014. Évaluation des éléments traces métalliques dans les fientes de volaille recouvrant les coquilles d'œufs destinés à la consommation humaine en Côte d'Ivoire. J. Appl. Biosci., 74: 60336042. http://dx.doi.org/10.4314/jab.v74i1.10

Anju T. 2012. Effect of Cadmium Acetate On Immuno- Hematological Parameters in White Leg-Horn Chicks. IAAST, 3(4): 40-42.

Bedii C, Kenan E. 2005. The Effects of Cadmium on Levels of Glucose in Serum and Glycogen Reserves in the Liver and Muscle Tissues of Cyprinus carpio (L., 1758). Turk J Vet Anim Sci., 29: 113-117.

Borane VR. 2013. Protective role of ascorbic acid on the cadmium induced changes in hematology of the freshwater fish, Channa orientalis (Schneider). Adv. Appl. Sci. Res., 4(2): 305-308.

Fahim MA, Nemmar A, Dhanasekaran S, Singh S, Shafiullah M, Yasin J, Zia S, Hasan MY. 2012. Acute Cadmium Exposure Causes Systemic and Thromboembolic Events in Mice. Physiol. Res., 61: 73-80.

Fawzia YS, Sayed GH, Walid SE, Hassan MD, Amira HM, Alaa RA. 2014. Protective Effects of Vitamin E, Selenium and Zinc Supplementation on Hematological and Some Biochemical Parameters in Pregnant Rats Exposed to 
Cadmium. Global J. Pharmacol., 8(4): 665-672.

Gabol K, Zaheer KM, Umair AKM, Peer K, Farina F, Saima S, Tanveer J, Nadeem B, Asif IM, Usman AHM, Muhammad T. 2014. Induced effects of lead, chromium and cadmium on gallus domesticus. Canadian Journal of Pure and Applied Sciences, 8(3): 3035-3042.

Haki K, Fikret K, Halit C. 2005. Effect of Single Dose Cadmium Chloride Administration on Oxidative Stress in Male and Female Rats. Turk J Vet Anim Sci., 29: 37-42.

Horiguchi H. 2007. Anemia induced by cadmium intoxication. Nihon Eiseigaku Zasshi. Japanese J. Hygien., 62(3): 888904.

Hounkpatin ASY, Edorh PA, Guédénon P, Alimba CG, Ogunkanmi A, Dougnon TV, Boni G, Aissi KA, Montcho S, Loko F, Ouazzani N, Mandi L, Boko M, Creppy EE. 2013. Haematological evaluation of Wistar rats exposed to chronic doses of cadmium, mercury and combined cadmium and mercury. Afr. J. Biotechnol., 12(23): 3731-3737. DOI: 10.5897/AJB12.2669

IARC. 1993. Cadmium and cadmium compounds. IARC Monographs on the Evaluation of Carcinogenic Risk of Chemicals to Humans, vol. 58. Lyon, France: International Agency for Research on Cancer. pp. 119-239.

Jelena D, Ivana M, Marina N, Aleksandra PA, Lidija Z, Dragan K, Milena K. 2014. Acute cadmium administration to rats exerts both immunosuppressive and proinflammatory effects in spleen. Toxicology, 326: 96-108. DOI : http://dx.doi.org/10.1016/j.tox.2014.10. 012

Koffi MK, Yolande AA, Justin SS, Henri MGAB. 2014. Evaluation de l'exposition de la population aux métaux traces (cadmium, mercure, plomb) à travers la consommation des viandes et abats de bœuf et de porc importés. Int. J. Biol. Chem. Sci., 8(4):
1594-1603.

DOI

http://dx.doi.org/10.4314/ijbcs.v8i4.21

Kouassi JK, Yves-Alain B, Ahoua ES, Denis B, Denezon OD, Moussa B, Fatiha Z, Peggy M. 2008. Diagnostic d'une contamination par les éléments traces métalliques de l'épinard (Spinacia Oleracea) Cultivé Sur des Sols Maraîchers de la Ville d'Abidjan (Côte d'Ivoire) amendés avec de la fiente de volailles. European Journal of Scientific Research, 21(3): 471-487.

Layachi N, Kechrid Z. 2012. Combined protective effect of vitamins $\mathrm{C}$ and $\mathrm{E}$ on cadmium induced oxidative liver injury in rats. African Journal of Biotechnology, 11(93): 16013-16020. DOI: $10.5897 / A J B 12.2665$

Lodia S, Kansala L. 2012. Antioxidant activity of Rubia cordifolia against lead toxicity. Int. J. Pharmacol. Sci. Res., 3(7): 2224-2232

Milovanovic A, Milovanovi A, Milovanovic J, Emerikic D, Novakovic M, Petrovic M, Jovanovic A, Petronic I. 2009. Effects of acute cadmium toxicity on oxidative damage in nervous tissue. Acta Veterinaria (Beograd), 59(5-6): 633-640. DOI: 10.2298/AVB0906633M

Madjouma K, Kpérkouma W, Komlan B, Gbandi DB, Adam A, Koffi A. 2009. Le maraîchage périurbain à Lomé : pratiques culturales, risques sanitaires et dynamiques spatiales. Cahiers Agricultures, 18(4): 356-363.

Nantarie T, Kouadio KP, Yoboue KE, YaoKouame A. 2015. Evaluation des métaux traces, pesticides et plastifiants dans les produits maraichers (Gombo, corète potagère, épinard et aubergine) dans la vallée du Nieki, sud-est de la Côte d'Ivoire. European Scientific Journal, 11(33): 183-196.

Oluwafemi AO, Basiru AA, Babatunji EO, Adebola BO. 2014. Hematological properties of Irvingia gabonensis in males adult rats. J. pharm Sci Innov, $\begin{array}{llll}3(5): & 434 & - & 436 .\end{array}$ http://dx.doi.org/10.7897/2277-4572.035190 
Othumpangat S, Kashon M, Joseph P. 2005. Eukaryotic translation initiation factor $4 \mathrm{E}$ is a cellular target for toxicity and death due to exposure to cadmium chloride. J. Biol. Chem., 280: 162-169.

Padmanaban AM, Mohan K. 2013. Toxic effects of cadmium chloride on hematological changes in freshwater field crab Paratelphusa hydrodromous (Decapoda: Brachyura). IJIRSET, 2(8): 3431-3435.

Satarug S, Moore MR. 2004. Adverse Health Effects of Chronic Exposure to LowLevel Cadmium in Foodstuffs and Cigarette Smoke. Environmental Health Perspectives, 112(10): 1099-1103.
Shim JY, Shin H, Han JG, Park HS, Lim BL, Chung KW, Om AS. 2008. Protective effects of Chlorella vulgaris on liver toxicity in cadmium-administered rats. $J$ Med Food, 11: 479-485.

Vinodini NA, Pratik KC, Poulomi C, Shrijeet C, Nayanatara AK, Ramesh MB, Rashmi KS, Suman VB, Sneha BS, Sheila RP. 2015. Protective Role of Aqueous Leaf Extract of Moringa oleiferaon Blood Parameters in Cadmium Exposed Adult Wistar Albino Rats. International Journal of Current Research and Academic Review, 3(1): 192-199. 